International Mathematical Forum, Vol. 8, 2013, no. 15, 743 - 751 HIKARI Ltd, www.m-hikari.com

\title{
Independent Transversal Equitable Domination in Graphs
}

\author{
Dhananjaya Murthy B. V ${ }^{1}$, G. Deepak ${ }^{1}$ and N. D. Soner ${ }^{2}$ \\ 1 Department of Mathematics
}

Nitte Meenakshi Institute of Technology, Yelahanka, Bangalore, India deepak1873@gmail.com

${ }^{2}$ Department of Studies in Mathematics

University of Mysore, Mysore 570 006, India

Copyright (c) 2013 Dhananjaya Murthy B. V et al. This is an open access article distributed under the Creative Commons Attribution License, which permits unrestricted use, distribution, and reproduction in any medium, provided the original work is properly cited.

\begin{abstract}
A set $S \subseteq V$ of vertices in a graph $G=(V, E)$ is called an equitable dominating set if for every vertex $u$ in $V-S$ there exists at least one vertex $v$ in $S$ adjacent to $u$ and $|\operatorname{deg}(u)-\operatorname{deg}(v)| \leq 1$. An equitable dominating set which intersects every maximum independent set in $G$ is called an independent transversal equitable dominating set. The minimum cardinality of an independent transversal equitable dominating set is called the independent transversal equitable domination number of $G$ and is denoted by $\gamma_{i t e}(G)$. In this paper we begin an investigation of this parameter.
\end{abstract}

\section{Mathematics Subject Classification: 05C69}

Keywords: Dominating set, equitable dominating set, independent transversal equitable dominating set, independent transversal equitable domination number

\section{Introduction}

By a graph $G=(V, E)$ we mean a finite and undirected graph with no loops and multiple edges. As usual $p=|V|$ and $q=|E|$ denote the number of vertices and edges of a graph $\mathrm{G}$, respectively. In general, we use $\langle X\rangle$ to denote 
the subgraph induced by the set of vertices $X . N(v)$ and $N[v]$ denote the open and closed neighbourhood of a vertex $v$, respectively. A set $D$ of vertices in a graph $G$ is a dominating set if every vertex in $V-D$ is adjacent to some vertex in $D$. The domination number $\gamma(G)$ is the minimum cardinality of a dominating set of $G$.

A line graph $L(G)$ (also called an interchange graph or edge graph) of a simple graph $G$ is obtained by associating a vertex with each edge of the graph and connecting two vertices with an edge if and only if the corresponding edges of $G$ have a vertex in common. A matching $M$ in $G$ is a set of pairwise nonadjacent edges; that is, no two edges share a common vertex. The matching number is the maximum cardinality of a matching of $G$ and is denoted by $\beta_{1}(G)$.

The independent transversal domination was introduced by I. S. Hamid [3]. A dominating set $D$ in a graph $G$ which intersects every maximum independent set in $G$ is called independent transversal dominating set of $G$. The minimum cardinality of an independent transversal dominating set is called independent transversal domination number of $G$ and is denoted by $\gamma_{i t}(G)$.

For terminology and notations not specifically defined here we refer reader to [4]. A subset $D$ of $V(G)$ is called an equitable dominating set of a graph $G$ if for every $u \in\langle V-D\rangle$, there exists a vertex $v \in D$ such that $u v \in E(G)$ and $|\operatorname{deg}(u)-\operatorname{deg}(v)| \leq 1$. The minimum cardinality of such a dominating set is denoted by $\gamma_{e}(G)$ and is called equitable domination number of $G$. $D$ is minimal if for any vertex $u \in D, D-\{u\}$ is not a equitable dominating set of $G$. A subset $S$ of $V$ is called a equitable independent set, if for any $u \in S, v \notin$ $N_{e}(u)$, for all $v \in S-\{u\}$. If a vertex $u \in V$ be such that $|\operatorname{deg}(u)-\operatorname{deg}(v)| \geq 2$ for all $v \in N(u)$ then $u$ is in each equitable dominating set. Such vertices are called equitable isolates. The equitable neighbourhood of $u$ denoted by $N_{e}(u)$ is defined as $N_{e}(u)=\{v \in V / v \in N(u),|\operatorname{deg}(u)-\operatorname{deg}(v)| \leq 1\}$ and $u \in I_{e} \Longleftrightarrow$ $N_{e}(u)=\emptyset$. The cardinality of $N_{e}(u)$ is denoted by $\operatorname{deg}_{e}(u)$. The maximum and minimum equitable degree of a point in $G$ are denoted respectively by $\Delta_{e}(G)$ and $\delta_{e}(G)$. That is $\Delta_{e}(G)=\max _{u \in V(G)}\left|N_{e}(u)\right|, \delta_{e}(G)=\min _{u \in V(G)}\left|N_{e}(u)\right|$. An edge $e=u v$ called equitable edge if $|\operatorname{deg}(u)-\operatorname{deg}(v)| \leq 1$. For more details about equitable domination number see[11]. .

\section{Independent Transversal equitable Domina- tion Number}

In this section, we determine the value of independent transversal equitable domination number for some standard families of graphs such as paths, cycles, complete bipartite and wheels. Also we determine $\gamma_{i t e}(G)$ for disconnected 
graphs.

Definition 2.1. Let $G=(V, E)$ be a graph. An equitable dominating set $S$ which intersects every maximum independent set in $G$ is called independent transversal equitable dominating set. The minimum cardinality of independent transversal equitable dominating set is called the independent transversal equitable domination number of $G$ and is denoted by $\gamma_{i t e}(G)$

Definition 2.2. An independent transversal equitable dominating set $D$ of a graph $G$ is said to be minimal independent transversal equitable dominating set if no proper subset of $D$ is independent transversal equitable dominating set.

Obviously any minimum independent transversal equitable dominating set $D$ is also minimal, but the converse is not true as illustrated in the next example.

Example 2.3. Let $G$ be a graph as in Figure 1.

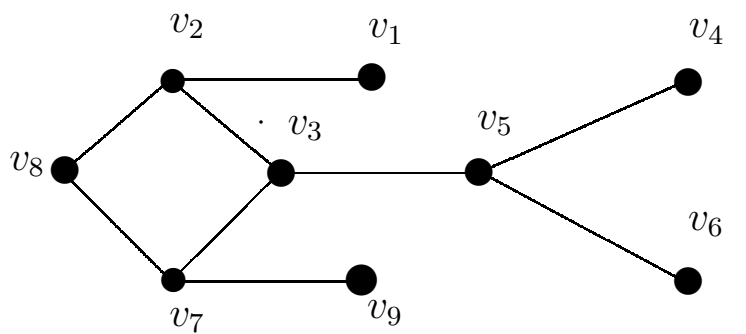

Figure 1: G

The domination number of the graph in Figure 1 is $\gamma(G)=3$, the minimum dominating sets are $\left\{v_{2}, v_{5}, v_{7}\right\},\left\{v_{2}, v_{5}, v_{8}\right\}$ and $\left\{v_{1}, v_{5}, v_{7}\right\}$.

There is only one maximum independent sets which is $\left\{v_{1}, v_{3}, v_{4}, v_{6}, v_{8}\right\}$. The independent transversal domination number of $G$ is $\gamma_{i t}(G)=3$, the sets $\left\{v_{2}, v_{5}, v_{8}\right\}$ and $\left\{v_{1}, v_{5}, v_{7}\right\}$ are minimum independent transversal dominating sets.

The independent transversal equitable domination number of $G$ is $\gamma_{i t e}(G)=$ 6 , the minimum independent transversal equitable dominating sets of $G$ are $\left\{v_{1}, v_{4}, v_{5}, v_{6}, v_{8}, v_{9}\right\},\left\{v_{1}, v_{3}, v_{4}, v_{6}, v_{8}, v_{9}\right\},\left\{v_{1}, v_{3}, v_{4}, v_{6}, v_{7}, v_{8}\right\}$ and $\left\{v_{1}, v_{2}, v_{3}, v_{4}, v_{6}, v_{8}\right\}$. The set $\left\{v_{1}, v_{2}, v_{4}, v_{5}, v_{6}, v_{7}, v_{8}\right\}$ is minimal independent transversal equitable dominating set of $G$ but not minimum.

If $G=(V, E)$ be any graph, then $V$ is independent transversal dominating set of $G$. Thus, the independent transversal domination number is defined for 
any graph $G$

The following inequality shows the relation between the domination number and degree equitable domination number an the independent transversal equitable domination number of a graph $G$.

Proposition 2.4. For any graph $G$,

$$
\gamma(G) \leq \gamma_{e}(G) \leq \gamma_{i t e}(G)
$$

Proof. Let $G$ be a graph with minimum independent transversal equitable dominating set $D$, then $D$ is an equitable dominating set of $G$ and any equitable dominating set is also dominating set of $G$ and from the definitions of the parameters $\gamma(G), \gamma_{e}(G)$ and $\gamma_{i t e}(G)$, we get $\gamma(G) \leq \gamma_{e}(G) \leq \gamma_{i t e}(G)$ for any graph $G$.

Proposition 2.5. For any graph $G$,

$$
\gamma(G) \leq \gamma_{i t}(G) \leq \gamma_{i t e}(G)
$$

Proof. Let $G$ be a graph with minimum independent transversal equitable dominating set $S$. From the definition of the independent transversal dominating set of $G, S$ is also independent transversal dominating set, and any independent transversal dominating set is dominating set of $G$. Hence $\gamma(G) \leq$ $\gamma_{i t}(G) \leq \gamma_{i t e}(G)$

A graph $G=(V, E)$ is called $(k ; k+1)$ bi-regular graph, if for any vertex $u \in V(G), \operatorname{deg}(u)=k$ or $k+1$ for some $k \geq 0$.

Theorem 2.6. [11] If $G$ is regular or $(k ; k+1)$ bi-regular graph, for some $k$, then $\gamma_{e}(G)=\gamma(G)$.

Theorem 2.7. If $G$ is regular or $(k ; k+1)$ bi-regular graph, for some $k \geq 0$, then $\gamma_{i t}(G)=\gamma_{i t e}(G)$.

Proof. Let $G$ be any regular graph and let $D$ be an independent transversal dominating set of $G$, such that $|D|=\gamma_{i t}(G)$. Then $D$ is dominating set which intersect every maximum independent set of $G$ and by theorem $2.6 \mathrm{D}$ is equitable dominating set intersect every maximum independent set of $G$. Therefore $\gamma_{i t e}(G) \leq \gamma_{i t}(G)$. Also by Proposition 2.5

$\gamma_{i t}(G) \leq \gamma_{i t}(G)$.

Hence If $G$ is regular graph, then $\gamma_{i t}(G)=\gamma_{i t e}(G)$.

Similarly by using Theorem 2.6 and Proposition 2.5 and by the same way we can prove that if $G$ is $(k ; k+1)$ bi-regular graph, for some $k \geq 0$, then $\gamma_{i t}(G)=\gamma_{i t e}(G)$.

We generalize the Theorem 2.6 by the following result. 
Theorem 2.8. For any graph $G$ which all of its edges are equitable, $\gamma_{i t}(G)=$ $\gamma_{\text {ite }}(G)$.

Proof. Let $G$ be a graph $G=(V, E)$ such that for any edge $e \in E e$ is equitable edge. Then any dominating set of $G$ will also be equitable dominating set of $G$. Suppose that $D$ is an independent transversal dominating set of $G$ with size $|D|=\gamma_{i t}(G)$. Then $D$ is also an independent transversal equitable dominating set, that means $\gamma_{i t}(G)=\gamma_{i t e}(G)$ and by Proposition 2.5, we have $\gamma_{i t}(G)=\gamma_{i t e}(G)$. Hence $\gamma_{i t}(G)=\gamma_{i t e}(G)$.

The converse of Theorem 2.8 is not true in general as illustrated in the following example.

Example 2.9. Let $G$ be a graph as in Figure 2, we have $\gamma_{i t}(G)=\gamma_{i t e}(G)=3$ the maximum independent sets in $G$ are $\left\{v_{1}, v_{4}, v_{5}\right\}$ and $\left\{v_{2}, v_{4}, v_{5}\right\}$. The minimum independent transversal dominating sets are $\left\{v_{1}, v_{4}, v_{5}\right\},\left\{v_{2}, v_{4}, v_{5}\right\}$ and $\left\{v_{1}, v_{2}, v_{3}\right\}$ and $\gamma_{i t}(G)=3$.

The minimum independent transversal equitable dominating set of $G$ is only $\left\{v_{2}, v_{4}, v_{5}\right\}$. So $\gamma_{i t e}(G)=3$. Hence $\gamma_{i t e}(G)=\gamma_{i t}(G)$, but the edge $v_{3} v_{4}$ and $v_{3} v_{5}$ are not equitable edges in $G$.

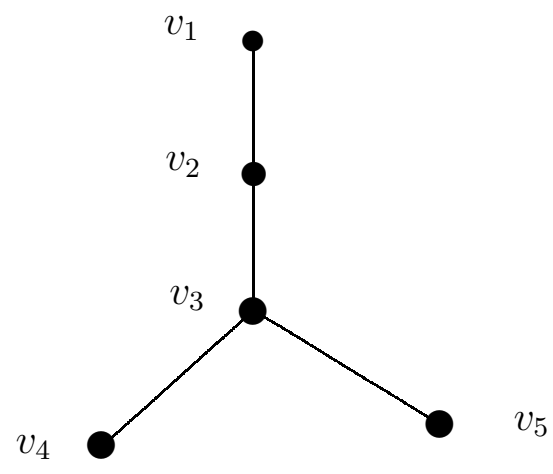

Figure 2: $\gamma_{i t}(G)=\gamma_{i t e}(G)$ but not all the edges are equitable edges

Theorem 2.10. [3] For any path $P_{p}$ with $p \geq 3$, we have

$$
\gamma_{i t}\left(P_{p}\right)= \begin{cases}2, & \text { if } p=2 \text { or } 3 \\ 3, & \text { if } p=6 \\ \left\lceil\frac{p-1}{3}\right\rceil, & \text { otherwise. }\end{cases}
$$

Theorem 2.11. [3] For any cycle $C_{p}$ of order $p$, we have

$$
\gamma_{i t}\left(C_{p}\right)= \begin{cases}3, & p=3,5 ; \\ \left\lceil\frac{p}{3}\right\rceil, & \text { otherwise. }\end{cases}
$$


From Theorem 2.10, Theorem 2.11 and Theorem 2.7 the following results are immediate.

Proposition 2.12. For any path $P_{p}$ with $p \geq 3$, we have

$$
\gamma_{i t e}\left(P_{p}\right)= \begin{cases}2, & \text { if } p=2 \text { or } 3 \\ 3, & \text { if } p=6 \\ \left\lceil\frac{p-1}{3}\right\rceil, & \text { otherwise. }\end{cases}
$$

Proposition 2.13. For any cycle $C_{p}$ of order $p$, we have

$$
\gamma_{i t e}\left(C_{p}\right)= \begin{cases}3, & p=3,5 \\ \left\lceil\frac{p}{3}\right\rceil, & \text { otherwise. }\end{cases}
$$

Theorem 2.14. Let $G=(V, E)$ be any graph with at least one isolated vertex, then $\gamma_{i t e}(G)=\gamma_{e}(G)$.

Proof. Let $G=(V, E)$ be a graph with at least one isolated vertex say $v$ and $S$ is a minimum equitable dominating set of $G$ that is $\gamma_{e}(G)=|S|$. Obviously the vertex $v$ must be belong to any equitable dominating set and also to any maximum independent set of $G$. Therefore the minimum equitable dominating set $S$ is is intersect every maximum independent set of $G$. Hence $S$ is minimum independent transversal equitable dominating set of $G$. Thus, $\gamma_{i t e}(G)=\gamma_{e}(G)$.

Theorem 2.15. For any graph $G=(V, E)$ with $p$ vertices, $1 \leq \gamma_{i t e}(G) \leq p$. Further $\gamma_{i t e}(G)=p$ if and only if either $G=K_{p}$ or for any vertex $v \in V(G)$, $\operatorname{deg}_{e}(v)=0$

Proof. If $G=(V, E)$ be any graph with $p$ vertices, then $V(G)$ is an independent transversal equitable dominating set of $G$. Therefore $\gamma_{i t e}(G) \leq p$ and also if the graph contains only one vertex we have $\gamma_{i t e}(G) \leq 1$. Hence $1 \leq \gamma_{i t e}(G) \leq p$.

If $G=K_{p}$, then clearly $\gamma_{i t e}(G) \leq p$, similarly if $G=(V, E)$ be a graph such that $v \in V(G)$, $\operatorname{deg}_{e}(v)=0$, then $\gamma_{e}(G)=p$ and since $\gamma_{e}(G) \leq \gamma_{i t e}(G)$. therefore $\gamma_{i t e}(G)=1$. Hence if $G=K_{p}$ or for any vertex $v \in V(G), \operatorname{deg}_{e}(v)=0$, then $\gamma_{i t e}(G)=p$.

Conversely,suppose that $\gamma_{i t e}(G)=p$, if $p=1$, then $G=K_{1}$, so let $\gamma_{i t e}(G)=p$ and $p \geq 2$. Now if the maximum equitable independence number $\beta_{e}(G) \geq 2$, then for some vertex $v \in V(G)$ we have $V(G)-\{v\}$ is independent transversal equitable dominating set, that means $\gamma_{i t e}(G) \leq p-1$ a contradiction. Therefore $\beta_{e}(G)=1$. Hence $G=K_{p}$ or for any vertex $v \in V(G), \operatorname{deg}_{e}(v)=0$.

Theorem 2.16. Let $G$ be a connected graph with $p$ vertices such that $\delta_{e}(G) \geq$ 1. Then $\gamma_{\text {ite }}(G)=p-1$ if and only if $G \cong P_{3}$. 
Proof. If $G \cong P_{3}$, then obviously $\gamma_{i t e}(G)=3$, that is $\gamma_{i t e}(G)=p-1$.

Conversely, suppose that $G$ be a connected graph with $p$ vertices such that $\delta_{e}(G) \geq 1$. Then the maximum equitable independence number $\beta_{e}(G) \geq 2$. Now suppose that there exist two equitable adjacent vertices $u$ and $v$ of degree two. Then the set $S=V(G)-\{u, v\}$ is an independent transversal equitable dominating set of $G$ and hence $\gamma_{i t e}(G) \leq p-2$, a contradiction. Hence for any equitable adjacent vertices $u$ and $v$ either $\operatorname{deg}_{e}(u)=1$ or $\operatorname{deg}_{e}(v)=1$ and $\gamma_{i t e}(G)=p-1$. Hence $G \cong P_{3}$.

There are many graphs of order $p$ other than $p_{3}$, see the following example.

Example 2.17. Let $G$ be graph as in Figure 3.

There is only two equitable dominating sets of $G,\left\{v_{1}, v_{2}, v_{3}, v_{4}, v_{5}, v_{6}, v_{8}\right\}$ and $\left\{v_{1}, v_{2}, v_{3}, v_{4}, v_{5}, v_{7}, v_{8}\right\}$.

There are two maximum independent sets $\left\{v_{1}, v_{2}, v_{3}, v_{4}, v_{5}, v_{6}\right\}$ and $\left\{v_{1}, v_{2}, v_{3}, v_{4}, v_{5}, v_{7}\right\}$. Therefore $\gamma_{e}(G)=\gamma_{i t e}(G)=7$. Hence $\gamma_{i t e}(G)=p-1$.

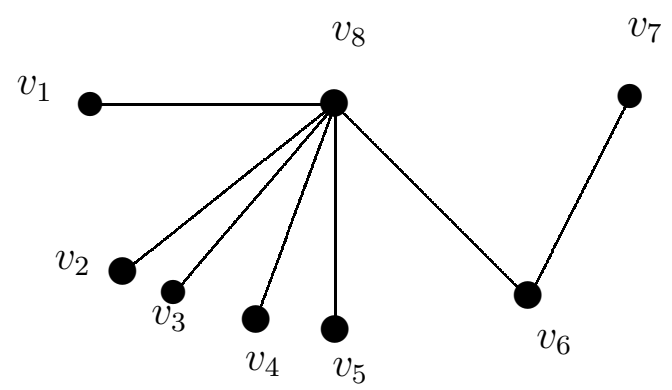

Figure 3: $\gamma_{i t}(G)=\gamma_{e}(G)=p-1$

Proposition 2.18. If $G$ be a graph of order $p$ such that $\gamma_{e}(G)=p-1$, then $\gamma_{i t}(G)=p-1$.

Proof. Suppose we have a graph $G$ with $p$ vertices and $\gamma_{e}(G)=p-1$, to prove that $\gamma_{i t}(G)=p-1$. Suppose that $\gamma_{i t}(G) \neq p-1$. Then by Proposition 2.4 $\gamma_{e}(G) \leq \gamma_{i t}(G)$ for any graph $G$. Therefore $\gamma_{i t}(G)=p$, then by Theorem2.15, $G$ is either $K_{p}$ or for any vertex $v \in V(G)$, $\operatorname{deg}_{e}(v)=0$, that means $\gamma_{e}(G)=p$, a contradiction. Hence $\gamma_{i t}(G)=p-1$.

Theorem 2.19. For any positive integer $a \geq 3$, there exist a graph $G$ of order $2 a$ and $\gamma_{i t e}(G)=a+1$.

Proof. Let $H$ be a complete graph of order $a \geq 3$. Let $G$ be the graph obtained from $H$ by attaching pendent edge in each vertex of $H$. Let $V(H)=$ 
$\left\{v_{1}, v_{2}, \ldots, v_{a}\right\}$ and let the pendent vertices are $u_{1}, u_{2}, \ldots, u_{a}$.

Now clearly any minimum equitable dominating set of $G$ contains all the pendent vertices $u_{1}, u_{2}, \ldots u_{a}$ and one vertex from the complete graph that the minimum dominating set of $G$ is of the form $u_{1}, u_{2}, \ldots u_{a}, v_{i}$ such that $i \in\{1,2, \ldots, a\}$ that is $\gamma_{e}(G)=a+1$, and there is only one maximum independent set $u_{1}, u_{2}, \ldots u_{a}$. Hence $\gamma_{i t e}(G)=\gamma_{e}(G)=a+1$.

Theorem 2.20. For any graph $G=(V, E), \gamma_{i t e}(G) \leq \delta_{e}(G)+\gamma_{e}(G)$.

Proof. Let $G=(V, E)$ be a graph and $v$ be any vertex in $V(G)$ such that $\operatorname{deg}_{e}(v)=\delta_{e}(G)$ and let $D$ be minimum equitable dominating set of $G$. Then every maximum independent set of $G$ contain a vertex of $N_{e}[u]$, so $D \cup N_{e}[u]$ is independent transversal equitable dominating set. We have also $S$ intersects $N_{e}[u]$. Therefore $\left|D \cup N_{e}[u]\right| \leq \delta_{e}(G)+\gamma_{e}(G)$. Hence $\gamma_{i t e}(G) \leq \delta_{e}(G)+\gamma_{e}(G)$.

Corollary 2.21. For any graph $G$ with equitable isolated vertex, $\gamma_{i t e}(G) \leq$ $\gamma_{e}(G)$.

Theorem 2.22. For any connected graph $G=(V, E)$ with $\delta_{e}(G)=1, \gamma_{i t e}(G) \leq$ $\frac{n+2}{2}$.

Proof. Let $G=(V, E)$ be any connected graph, with $\delta_{e}(G)=1$. Then by Theorem 2.20. $\gamma_{i t e}(G) \leq \gamma_{e}(G)+1$. and from [11] if $G$ has no equitable isolated vertices, we have $\gamma_{e} \leq \frac{\bar{p}}{2}$. Hence $\gamma_{i t e}(G) \leq \frac{p+2}{2}$.

\section{Acknowledgement}

The first author express his deep sense of gratitude and appreciation to the Director, N. R. Shetty, Nitte Meenakshi Institute of Technology, Yelahanka, Bangalore for his constant support and encouragement for research and development.

\section{References}

[1] J. Bondy and U. Murthy, Graph Theory with applications, North Holland, New York, (1976).

[2] C. Godsil and G. Royle, Algebraic graph theory, vol. 207 of Graduate Texts in Mathematics, Springer-Verlag, New York, 2001.

[3] I. S. Hamid, Independent transversal Domination in Graphs,Discussions Mathematicae Graph Theory 32(2012) 5-17.

[4] F. Harary, Graph theory, Addison-Wesley, Reading Mass (1969).

[5] T. W. Haynes, S. T. Hedetniemi and P. J. Slater, Fundamentals of domination in graphs, Marcel Dekker, Inc., New York (1998). 
[6] S. M. Hedetneimi, S. T. Hedetneimi, R. C. Laskar, L. Markus and P. J. Slater. Disjoint dominating sets in graphs. Proc. Int. Conf. on Disc.Math., IMI-IISc, Bangalore (2006) 88 - 101.

[7] S. R. Jayaram, Line domination in graphs, Graphs Combin. 3 (1987), 357-363.

[8] V. R. Kulli and S. C. Sigarkanti, Further results on the neighborhood number of a graph. Indian J. Pure and Appl. Math.23 (8) (1992) 575 -577 .

[9] S. Mitchell and S. T. Hedetniemi, Edge domination in trees, Congr. Numer.19 (1977), 489-509.

[10] E. Sampathkumar and P. S. Neeralagi, The neighborhood number of a graph, Indian J. Pure and Appl. Math.16 (2) (1985) 126 - 132.

[11] Venkatasubramanian Swaminathan And Kuppusamy Markandan Dharmalingam, Kragujevac Journal of Mathematics, Degree Equitable Domination On Graphs, Volume 35 Number 1 (2011), Pages 191-197.

[12] H. B. Walikar, B. D. Acharya and E. Sampathkumar, Recent developmentsin the theory of domination in graphs, Mehta Research instutute, Allahabad, MRI Lecture Notes in Math. 1 (1979).

\section{Received: February 14, 2013}

